\title{
ROOM TEMPERATURE OpERATION OF GASB-BASED RESONANT TUNNELING DIODES By PREWELL INJECTION
}

\author{
Andreas Pfenning, ${ }^{1, a)}$ Georg Knebl, ${ }^{1}$ Fabian Hartmann, ${ }^{1, b)}$ Robert Weih, ${ }^{1}$ Andreas Bader, ${ }^{1}$ \\ Monika Emmerling, ${ }^{1}$ Martin Kamp, ${ }^{1}$ Sven Höfling, ${ }^{1,2}$ Lukas Worschech ${ }^{1}$ \\ 1) Technische Physik, Physikalisches Institut and Röntgen Center for Complex Material Systems (RCCM), Universität \\ Würzburg, Am Hubland, D-97074 Würzburg, Germany \\ ${ }^{2)}$ SUPA, School of Physics and Astronomy, University of St. Andrews, St. Andrews, KY16 9SS, United Kingdom
}

We present room temperature resonant tunneling of GaSb/AIAsSb double barrier resonant tunneling diodes with pseudomorphically grown prewell emitter structures comprising the ternary compound semiconductors GalnSb and GaAsSb. At room temperature, resonant tunneling is absent for diode structures without prewell emitters. The incorporation of $\mathrm{Ga}_{0.84} \mathrm{In}_{0.16} \mathrm{Sb}$ and $\mathrm{GaAs}_{0.05} \mathrm{Sb}_{0.95}$ prewell emitters leads to room temperature resonant tunneling with peak-to-valley current ratios of 1.45 and 1.36 , respectively. The room temperature operation is attributed to the enhanced $\Gamma$-L-valley energy separation and consequently depopulation of L-valley states in the conduction band of the ternary compound emitter prewell with respect to bulk GaSb.

a) Electronic mail to: Andreas.Pfenning@physik.uni-wuerzburg.de
b) Electronic mail to: Fabian.Hartmann@physik.uni-wuerzburg.de

The three semiconductors GaSb, InAs and AISb of the so-called $6.1 \AA$ family cover a wide range of bandgap energies and unique material properties, which make them particularly suitable for applications in high-speed electronics and as mid-infrared optoelectronic semiconductor devices. ${ }^{1,2}$ During the past few years, application related research focused on mid-infrared light sources and detectors. ${ }^{3,4}$ Especially the progress on interband cascade lasers (ICLs) and interband cascade detectors (ICDs) with type-II superlattice absorbers has driven the field. ${ }^{5-7}$ In a recent publication we proposed an alternative mid-infrared photodetector concept based on resonant tunneling diodes (RTDs) with $6.1 \AA$ family semiconductors. ${ }^{8}$ RTDs can be exploited as highspeed and low-noise amplifiers of weak, optically excited electrical signals. ${ }^{9-11}$ Unlike avalanche photodiodes, in which the multiplication gain originates from impact ionization, the RTD photodetection principle is based on the modulation of the resonant tunneling current via Coulomb interaction in presence of photogenerated minority charge carriers. ${ }^{12-14}$ This mechanism provides very high amplification factors exceeding several hundred thousand at considerably low operation voltages. ${ }^{10,11,15,16}$ The GaSb/InAs/AISb material system has brought forth resonant tunneling structures (RTS) with unique and enhanced characteristics. ${ }^{2,17}$ Resonant interband tunneling diodes (RITDs) for example exploit the broken bandgap alignment of GaSb and InAs, which leads to considerably low resonance voltages as low as $90 \mathrm{mV} .^{18}$ Bandgap blocking at bias voltages exceeding the resonance voltage ensures high room temperature peak-to-valley current ratios well above $P V C R>$ $20 .^{19,20}$ RTDs employing an AISb double barrier structure with InAs quantum wells and contacts have mainly 
been studied for high speed switching with oscillator frequencies up to $f=712 \mathrm{GHz} .^{21,22}$ Despite all these desirable properties (low voltage, high PVCR and high speed), most RTDs and RITDs of the $6.1 \AA$ family are poorly suited as photodetectors, since the staggered or even broken type-Il bandgap alignment does not provide the means for sufficient accumulation of minority charge carriers at the RTS. The AlSb/GaSb double barrier RTS resembles conventional AIGaAs/GaAs RTDs and provides a well-defined type-I band alignment with large offsets in conduction and valence band. ${ }^{23}$ Surprisingly, GaSb/AIGaSb double barrier RTS have been hardly investigated. Although resonant tunneling has been observed at cryogenic temperatures, it has not yet been demonstrated at room temperature. This can most likely be attributed to the small $\Gamma$-L-valley energy separation within GaSb. ${ }^{23}$

In this publication, we investigate the electronic transport properties of AISb/GaSb RTDs with pseudomorphically grown ternary emitter prewells. The main purpose of the emitter prewells is to increase the $\Gamma$-L-valley energy separation and thus to suppress L-valley and $\Gamma$-L-valley transport channels. Additionally, an enhancement of RTD transport properties can be expected in case of sufficiently deep prewells, due to quantization effects. ${ }^{24-26}$ For structures without a prewell emitter, resonant tunneling and peak-to-valley current ratios exceeding 8.2 are observed at a temperature of $77 \mathrm{~K}$. At room temperature, resonant tunneling is absent for diode structures without prewell emitters. The incorporation of $\mathrm{Ga}_{0.84} \mathrm{In}_{0.16} \mathrm{Sb}$ and $\mathrm{GaAs}_{0.05} \mathrm{Sb}_{0.95}$ prewell emitters leads to room temperature resonant tunneling with peak-to-valley current ratios of 1.45 and 1.36 , respectively.

The investigated samples RTD 1, RTD 2 and RTD 3 are grown by molecular beam epitaxy (MBE) on an ntype Te-doped GaSb (100) substrate. The MBE chamber is equipped with solid state evaporation cells for the group-III elements ( $\mathrm{Al}, \mathrm{Ga}, \mathrm{In}$ ) and dopants (Be, Si, Te). Group-V elements (As, Sb) are provided by valved cracking cells. During the growth process, the substrate temperature is controlled by a pyrometer. Prior to the growth, oxide desorption is realized under Sb-stabilization flux at a substrate temperature of $T=580{ }^{\circ} \mathrm{C}$. The layer structures of all three samples are provided in Figure 1 (a). First, 300 nm of n-type Te-doped GaSb are grown with $n=1 \times 10^{18} \mathrm{~cm}^{-3}$, followed by a $120 \mathrm{~nm}$ wide GaSb layer with a reduced doping concentration of $n=5 \times 10^{17} \mathrm{~cm}^{-3}$. The undoped resonant tunneling structure is grown subsequently, starting with $20 \mathrm{~nm}$ GaSb, followed by the $7 \mathrm{~nm}$ wide emitter prewell, and two $4.5 \mathrm{~nm}$ lattice matched $\mathrm{AlAs}_{0.08} \mathrm{Sb}_{0.92}$ barriers sandwiching a $5 \mathrm{~nm}$ quantum well. The three samples differ in the composition of the prewell emitters and quantum wells. For the reference sample (RTD 1), prewell and quantum well consist of the binary GaSb. For the samples RTD 2 and RTD 3, the ternary and pseudomorphically grown $\mathrm{Ga}_{0.84} \mathrm{In}_{0.16} \mathrm{Sb}$ and $\mathrm{GaAs}_{0.05} \mathrm{Sb}_{0.95}$ are employed, respectively. The resonant tunneling structure is finalized by $27 \mathrm{~nm}$ of undoped GaSb, followed by $120 \mathrm{~nm}$ of GaSb with a doping concentration of $n=5 \times 10^{17} \mathrm{~cm}^{-3}$ and $220 \mathrm{~nm}$ lattice-matched $\mathrm{Al}_{0.3} \mathrm{Ga}_{0.7} \mathrm{As}_{0.03} \mathrm{Sb}_{0.97}$ with a doping concentration of $n=1 \times 10^{18} \mathrm{~cm}^{-3}$. On top, a $10 \mathrm{~nm}$ GaSb capping layer with $n=1 \times 10^{18} \mathrm{~cm}^{-3}$ is deposited. The doping profiles are examined and confirmed by time-of-flight secondary ion mass spectroscopy (TOF-SIMS). Growth rates and width of the different semiconductor layer structures are examined and confirmed with cross-sectional scanning electron microscopy (SEM). Figure 1(b) shows an SEM image of the layer structure of RTD 1. Dark contrast represents regions with higher Al concentrations, which 
allows for identifying width and position of the AIAsSb double barrier structure as well as the AlGaAsSb contact region. The SEM images indicate good crystal quality with no apparent dislocations or defects.

Figure 1(c) shows a sketch of the conduction band (CB) profiles of the resonant tunneling structure of RTD 2 for the $\Gamma$ - and L-valley as solid black and red dashed line, respectively. Due to the small $\Gamma$ - $L$ valley energy separation in GaSb of only $\Delta E_{\Gamma-\mathrm{L}} \approx 26 \mathrm{meV}$ and the relatively low density of states at the $\Gamma$-point (compared to the L-valley), a significant fraction of electrons occupy L-valley states at room temperature. ${ }^{27}$ The ternary prewell lowers the $\Gamma$-, while increasing the L-valley. Hence, L-valley states in the emitter are depopulated.

Figure $1(\mathrm{~d})$ and (e) show the $\mathrm{CB}$ offset $\Delta E_{C}$ and the $\Gamma$-L valley energy separation $\Delta E_{\Gamma-\mathrm{L}}$ as a function of In and As mole fraction. $\Delta E_{C}$ and $\Delta E_{\Gamma-\mathrm{L}}$ are calculated using the material parameters provided in Ref. [27] The black and red arrow indicate the mole fraction used in RTD 2 and RTD 3, respectively. The mole fractions of 16 $\% \ln \left(\right.$ RTD 2) and $5 \%$ As (RTD 3) are chosen to provide comparable $\Delta E_{C}$ and $\Delta E_{\Gamma-\mathrm{L}}$ values.

High-resolution X-ray diffraction (HR-XRD) measurements confirm the mole fractions of the ternary and quaternary semiconductor layers. The experimental HR-XRD scans (black line) and the corresponding simulations (green line) are shown in Figure 2(a), (b) and (c) for RTD 1, RTD 2 and RTD 3, respectively. XRD counts are plotted versus the diffraction angle $\Delta \omega$, with the GaSb peak diffraction angle positioned at $\Delta \omega=0^{\circ}$. The HR-XRD spectrum of RTD 1 shows a single peak at $\Delta \omega=0^{\circ}$, which indicates a good and high quality latticematched crystal growth of the RTD and the AIGaAsSb contact region. For RTD 2 and RTD 3, compressive and tensile strain secondary patterns arise at smaller and higher angles, respectively, caused by the incorporation of the pseudomorphically GalnSb and GaAsSb regions. The secondary pattern of RTD 2 is more pronounced compared to RTD 3 due to the three times higher In compared to As concentration.

Circular RTD mesa structures with diameters from $d=2 \mu \mathrm{m}$ up to $d=13 \mu \mathrm{m}$ are defined by optical lithography and dry-chemical etching. The etching depth is about $50 \mathrm{~nm}$ below the double barrier structure. The polymer BCB ( $\underline{B} e n z o \underline{C} y c l o \underline{B} u t e n e)$ is used for mesa sidewall passivation. No further surface passivation treatments are applied. The RTD mesa structures are contacted with AuGe/Ni/Au on the substrate side and $\mathrm{Ti} / \mathrm{Au}$ ring-shaped contacts are deposited on top of the mesa.

Figure 3 shows the current density-voltage $(j(V))$-characteristics of the three RTD samples, measured at room temperature $(T=300 \mathrm{~K}$, red) and at $T=77 \mathrm{~K}$ (blue). For RTD 1 and at $77 \mathrm{~K}$ (Figure 3 (a)), a typical RTD characteristic with a well-pronounced current bistability is observed. The resonance occurs at a bias voltage of $V=2.1 \mathrm{~V}$ with a current density of $j_{\text {res }}=2.98 \mu \mathrm{A} / \mu \mathrm{m}^{2}$, and the valley current density is $j_{\text {val }}=0.36 \mu \mathrm{A} / \mu \mathrm{m}^{2}$. The corresponding peak-to-valley current ratio is $P V C R=8.2$, which is slightly above the previously reported value of $P V C R=7.6$ in a similar structure. ${ }^{23}$ According to Jimenez et al., the observed bistability is of intrinsic nature. ${ }^{28,29}$ For negative bias voltages (not shown here), identical resonance and valley current density values are observed. Hence, the RTS is of high quality and symmetry. At room temperature however, the current resonance disappears and only a little shoulder remains at about $V=1.5 \mathrm{~V}$, as indicated by the red arrow. At room temperature and due to the small $\Delta E_{\Gamma-\mathrm{L}}$ separation of GaSb, a significant fraction of electrons in the conduction band populate the L- instead of the $\Gamma$-point, ${ }^{27}$ and several additional transmission paths need to be 
considered, which in sum lead to a disappearance of the current resonance at room temperature. ${ }^{23}$ The $j(V)$ characteristics of RTD 2 and RTD 3 are sown in Figure 3 (b) and (c), respectively. Similar to RTD 1, both prewell samples show a well-pronounced resonance located at $V=1.4 \mathrm{~V}$ at a temperature of $T=77 \mathrm{~K}$, with peak-tovalley current ratios of $P V C R=7.8$ (RTD 2) and $P V R=4.0$ (RTD 3). In contrast to RTD 1, the valley regions of RTD 2 and 3 are much more pronounced and extend over a broader voltage region of about $0.5 \mathrm{~V}$ from $V \approx 1.5$ $\mathrm{V}$ up to $V \approx 2.0 \mathrm{~V}$. The lower PVCR of RTD 3 is caused by a reduced resonance current density $j_{\text {res }}=2.55$ $\mu \mathrm{A} / \mu \mathrm{m}^{2}$ and an increased valley current density $j_{v a l}=0.64 \mu \mathrm{A} / \mu \mathrm{m}^{2}$.

At room temperature, resonant tunneling with a well-pronounced current resonance and a negative differential conductance region is observed (RTD 2 and RTD 3). For both samples, the resonance is observed at values slightly below $V=1.1 \mathrm{~V}$. The corresponding peak-to-valley current ratios are $P V C R=1.4$ (RTD 2) and $P V C R=1.3\left(\right.$ RTD 3) with $j_{r e s}=2.12 \mu \mathrm{A} / \mu^{2}$ and $j_{r e s}=2.09 \mu \mathrm{A} / \mu \mathrm{m}^{2}$, respectively. Several conclusions can be drawn from a comparison of the $j(V)$-characteristics shown in Figure 3. Most importantly, the room temperature transport properties of GaSb-based double barrier resonant tunneling diodes can be significantly enhanced by incorporation a pseudomorphic prewell and hence, resonant tunneling at room temperature can be achieved. The improvement is only observed for prewell injection (positive bias voltages), whereas injection from bulk GaSb into the inter-barrier quantum well (negative bias voltages) shows no notable effect, as can be seen from the inset in Figure 3 (b). In GaAs RTDs with GalnAs prewells, an enhanced PVCR can be observed, mainly due to larger resonance currents, which originate from increased emitter charge carrier density, stronger 2D-2D tunneling and an enhanced wavefunction overlap. ${ }^{25,26,30-33}$ Since this is true at cryogenic temperatures as well as at room temperature, an increased resonance current should be apparent at any temperature. In the samples RTD 2 and RTD 3, the opposite is the case. On the contrary, the resonance current densities of RTD 2 and RTD 3 are reduced at $T=77 \mathrm{~K}$ compared to RTD 1, which suggests that the room temperature operation cannot be attributed solely to quantization effects in the prewell. Given the shallow nature of the prewells, this seems reasonable and it leaves the increased $\Gamma-L$ valley energy separation within the prewell as main contributing factor.

Surface leakage and reproducibility are important issues in Antimonide-based electronic devices that originate from the reactive antimonide surface. ${ }^{34}$ When exposed to an oxygen-containing environment such as air, the GaSb surface will form a native oxide layer and free Sb. ${ }^{35}$ Both the oxide layer and free Sb strongly diminish the device performance. While the free Sb can act as metallic low resistance shunt, the oxide layer creates additional interface states promoting generation-recombination centers, charge accumulation centers, or trap levels. ${ }^{34}$ Hence, a lot of effort is devoted to developing passivation techniques, such as dielectric passivation, ${ }^{36}$ chalcogenide passivation, ${ }^{37}$ or high-bandgap semiconductor overgrowth. ${ }^{38}$ To investigate surface leakage and reproducibility in our samples, we measure a series of room temperature I(V)-characteristics of RTD 2 and RTD 3 for diode diameters from $d=4 \mu \mathrm{m}$ up to $d=13 \mu \mathrm{m}$. The corresponding resonance $\left(I_{\text {res }}\right.$ black spheres) and valley currents ( $I_{v a l}$, red spheres) are shown in Figure $4(\mathrm{a})$ and (b) as a function of the diode area for RTD 2 and RTD 3, respectively. For both samples resonance and valley current increase linearly with the diode area and the slopes of the linear fits provide the current densities, with $j_{\text {res }}=2.13 \mu \mathrm{A} / \mu \mathrm{m}^{2}$ for RTD 2 and $j_{\text {res }}=2.01 \mu \mathrm{A} / \mu^{2}$ for RTD 3. The valley current densities are $j_{v a l}=1.47 \mu \mathrm{A} / \mu \mathrm{m}^{2}$ (RTD 2) and $j_{v a l}=1.48$ 
$\mu A / \mu m^{2}$ (RTD 3). The corresponding PVCRs are 1.45 and1.36 for RTD 2 and RTD 3, respectively. The PVCR obtained this way represents the PVCR of large scale RTDs. Notably for both samples, the linear fit function of $I_{r e s}$ and $I_{v a l}$ intersect the $\mathrm{x}$-axis at positive diode areas of $8 \mu \mathrm{m}^{2}$ and $7 \mu \mathrm{m}^{2}$, respectively. Hence, a distinct nonconductive region of about $1.5 \mu \mathrm{m}$ thickness is observed, which indicates that side-wall leakage is negligible. The non-conductive region can be caused by different mechanisms such as for example by a Fermi-level pinning, defects from the dry-chemical etching process, or oxidation of Al-rich layers. ${ }^{39,40}$ Even though surface Fermi-level pining in GaSb occurs in vicinity of the valence band edge, a depletion region as large as $1.5 \mu \mathrm{m}$ due to surface Fermi-level pinning does not seem reasonable. Thermal oxidation of AIAsSb is known to occur under heating conditions (e.g. baking process of the $\mathrm{BCB}$ ) and could explain the outer non-conductive region. ${ }^{40}$

In conclusion, we have demonstrated room temperature resonant tunneling of electrons in AlSb/GaSb double barrier resonant tunneling diodes by utilizing pseudomorphically grown ternary GalnSb and GaAsSb emitter prewells. Peak-to-valley current ratios of 1.45 (RTD 2, GalnSb) and 1.36 (RTD 3) have been observed. The improved room temperature transport properties result from a depopulation of L-valley states in the emitter prewell with respect to bulk GaSb. Further increasing the In or As mole fractions will supposedly result in a significant increase of the PVCR.

\section{Acknowledgements}

The authors are grateful for financial support by the state of Bavaria, the German Ministry of Education and Research (BMBF) within the national project HIRT (FKZ 13XP5003B). 


\section{References}

${ }^{1}$ H. Kroemer, Phys. E Low-Dimensional Syst. Nanostructures 20, 196 (2004).

${ }^{2}$ B.R. Bennett, R. Magno, J.B. Boos, W. Kruppa, and M.G. Ancona, Solid. State. Electron. 49, 1875 (2005).

${ }^{3}$ A. Bauer, K. Rößner, T. Lehnhardt, M. Kamp, S. Höfling, L. Worschech, and A. Forchel, Semicond. Sci. Technol. 26, 14032 (2010).

${ }^{4}$ P. Martyniuk, J. Antoszewski, M. Martyniuk, L. Faraone, and A. Rogalski, Appl. Phys. Rev. 1, 41102 (2014).

${ }^{5}$ R. Weih, L. Nähle, S. Höfling, J. Koeth, and M. Kamp, Appl. Phys. Lett. 105, 71111 (2014).

${ }^{6}$ I. Vurgaftman, R. Weih, M. Kamp, J.R. Meyer, C.L. Canedy, C.S. Kim, M. Kim, W.W. Bewley, C.D. Merritt, J. Abell, and S. Höfling, J. Phys. D. Appl. Phys. 48, 123001 (2015).

${ }^{7}$ R.Q. Yang, Z. Tian, Z. Cai, J.F. Klem, M.B. Johnson, and H.C. Liu, J. Appl. Phys. 107, 54514 (2010).

${ }^{8}$ S. Höfling, A. Pfenning, R. Weih, A. Ratajczak, F. Hartmann, G. Knebl, M. Kamp, and L. Worschech, in edited by M. Strojnik (2016), p. 997306.

${ }^{9}$ B. Romeira, L.M. Pessoa, H.M. Salgado, C.N. Ironside, and J.M.L. Figueiredo, Sensors (Basel). 13, 9464 (2013).

${ }^{10}$ A. Pfenning, F. Hartmann, F. Langer, S. Höfling, M. Kamp, and L. Worschech, Appl. Phys. Lett. 104, 101109 (2014).

${ }^{11}$ W. Wang, Y. Hou, D. Xiong, N. Li, W. Lu, W. Wang, H. Chen, J. Zhou, E. Wu, and H. Zeng, Appl. Phys. Lett. 92, 23508 (2008).

${ }^{12}$ P. England, J.E. Golub, L.T. Florez, and J.P. Harbison, Appl. Phys. Lett. 58, 887 (1991).

${ }^{13}$ P.W. Park, H.Y. Chu, S.G. Han, Y.W. Choi, G. Kim, and E.-H. Lee, Appl. Phys. Lett. 67, 1241 (1995).

${ }^{14}$ I.J.S. Coêlho, J.F. Martins-Filho, J.M.L. Figueiredo, and C.N. Ironside, J. Appl. Phys. 95, 8258 (2004).

${ }^{15}$ A. Pfenning, F. Hartmann, M. Rebello Sousa Dias, F. Langer, M. Kamp, L.K. Castelano, V. Lopez-Richard, G.E. Marques, S. Höfling, and L. Worschech, Appl. Phys. Lett. 107, 81104 (2015).

${ }^{16}$ A. Pfenning, F. Hartmann, F. Langer, M. Kamp, S. Höfling, and L. Worschech, Nanotechnology 27, 355202 (2016).

${ }^{17}$ G. Haddad, P. Mazumder, and J.N. Schulman, Proc. IEEE 86, 641 (1998).

${ }^{18}$ D. McMorrow and R. Magno, Nucl. Sci. ... 48, 1973 (2001).

${ }^{19}$ R. Magno, A.S. Bracker, and B.R. Bennett, J. Appl. Phys. 89, 5791 (2001).

${ }^{20}$ K.F. Longenbach, L.F. Luo, S. Xin, and W.I. Wang, J. Cryst. Growth 111, 651 (1991).

${ }^{21}$ E.R. Brown, J.R. Söderström, C.D. Parker, L.J. Mahoney, K.M. Molvar, and T.C. McGill, Appl. Phys. Lett. 58, 2291 (1991).

${ }^{22}$ D. Woolard, W. Zhang, and B. Gelmont, Solid. State. Electron. 49, 257 (2005).

${ }^{23}$ J.L. Jimenez, X. Li, and W.I. Wang, Appl. Phys. Lett. 64, 2127 (1994).

${ }^{24}$ T.B. Boykin, R.C. Bowen, G. Klimeck, and K.L. Lear, Appl. Phys. Lett. 75, 1302 (1999).

${ }^{25}$ R.M. Lewis, H.P. Wei, S.Y. Lin, and J.F. Klem, Appl. Phys. Lett. 77, 2722 (2000).

${ }^{26}$ H. Brugger, U. Meiners, C. Wölk, R. Deufel, A. Marten, M. Rossmanith, K. v. Klitzing, and R. Sauer, Microelectron. Eng. 15, 663 (1991).

${ }^{27}$ I. Vurgaftman, J.R. Meyer, and L.R. Ram-Mohan, J. Appl. Phys. 89, 5815 (2001).

${ }^{28}$ J. Jimenez, E. Mendez, X. Li, and W. Wang, Phys. Rev. B 52, (1995).

${ }^{29}$ J. Jimenez, E. Mendez, X. Li, and W. Wang, Solid. State. Electron. 40, 583 (1996).

${ }^{30}$ T.B. Boykin, R.C. Bowen, G. Klimeck, and K.L. Lear, Appl. Phys. Lett. 75, 1302 (1999).

${ }^{31}$ C.R. Wie and Y.W. Choi, Appl. Phys. Lett. 58, 1077 (1991).

${ }^{32}$ Y.W. Choi and C.R. Wie, J. Appl. Phys. 71, 1853 (1992). 
${ }^{33}$ R. Kapre, A. Madhukar, K. Kaviani, S. Guha, and K.C. Rajkumar, Appl. Phys. Lett. 56, 922 (1990).

${ }^{34}$ F.M. Mohammedy and M. Jamal Deen, J. Mater. Sci. Mater. Electron. 20, 1039 (2009).

${ }^{35}$ M. Pérotin, P. Coudray, L. Gouskov, H. Luquet, C. Llinarès, J.J. Bonnet, L. Soonckindt, and B. Lambert, J. Electron. Mater. 23, 7 (1994).

${ }^{36}$ A. Hood, M. Razeghi, E.H. Aifer, and G.J. Brown, Appl. Phys. Lett. 87, 151113 (2005).

${ }^{37}$ A. Gin, Y. Wei, A. Hood, A. Bajowala, V. Yazdanpanah, M. Razeghi, and M. Tidrow, Appl. Phys. Lett. 84, 2037 (2004).

${ }^{38}$ R. Rehm, M. Walther, F. Fuchs, J. Schmitz, and J. Fleissner, Appl. Phys. Lett. 86, 173501 (2005).

${ }^{39}$ P.S. Dutta, H.L. Bhat, and V. Kumar, J. Appl. Phys. 81, 5821 (1997).

${ }^{40}$ K. Meneou, J. Appl. Phys. 95, 5131 (2004). 
Figure 1: (a) Layer structures of the three resonant tunneling diodes: RTD 1, RTD 2 and RTD 3. (b) Cross-sectional scanning electron microscopy image of reference sample (RTD 1). Dark contrast regions correspond to Al-containing regions. (c) Conduction band profiles of the $\Gamma$-and L-points of sample RTD 2 with GalnSb quantum wells. (d) Conduction band offset (quantum well depth) as a function of In and As mole fractions. (e) Conduction band energy separation of $\Gamma$-and L-point as a function of In and As mole fraction. The black and red arrow indicate the mole fraction used in RTD 2 and RTD 3, respectively.

Figure 2: High-resolution X-ray diffraction (HR-XRD) spectra of RTD 1 (in a), RTD 2 (in b) and RTD 3 (in C). Experimental data is shown as solid black line and the simulated spectra are shown as solid green line. The HR-XRD spectra are centered at the GaSb substrate peak at $\Delta \omega=0^{\circ}$. For RTD 2 and RTD 3, tensile and compressive strain secondary patterns arise at smaller and higher angles, respectively.

Figure 3: Current density-voltage characteristics measured at room temperature $(T=300 \mathrm{~K}$, red) and at $T=77 \mathrm{~K}$ (blue) for the three RTD samples: (a) RTD 1 reference sample. (b) RTD 2 with $G a_{0.84} / n_{0.16} S b$ prewell and quantum well. The inset shows the current-density for negative bias voltages (bulk injection). (c) RTD 3 with GaAs ${ }_{0.05} S b_{0.05}$ prewell and quantum wells.

Figure 4: Resonance (black spheres) and valley current (red spheres) as a function of diode area for RTD 2 (in (a)) and RTD 3 (in (b)). From the slope of the linear fit functions, the ideal peak-to-valley current ratios of large scale diodes can be calculated. 


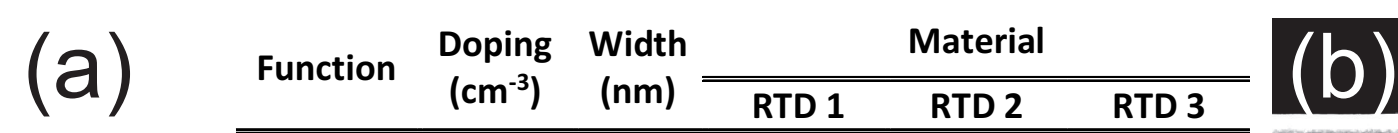

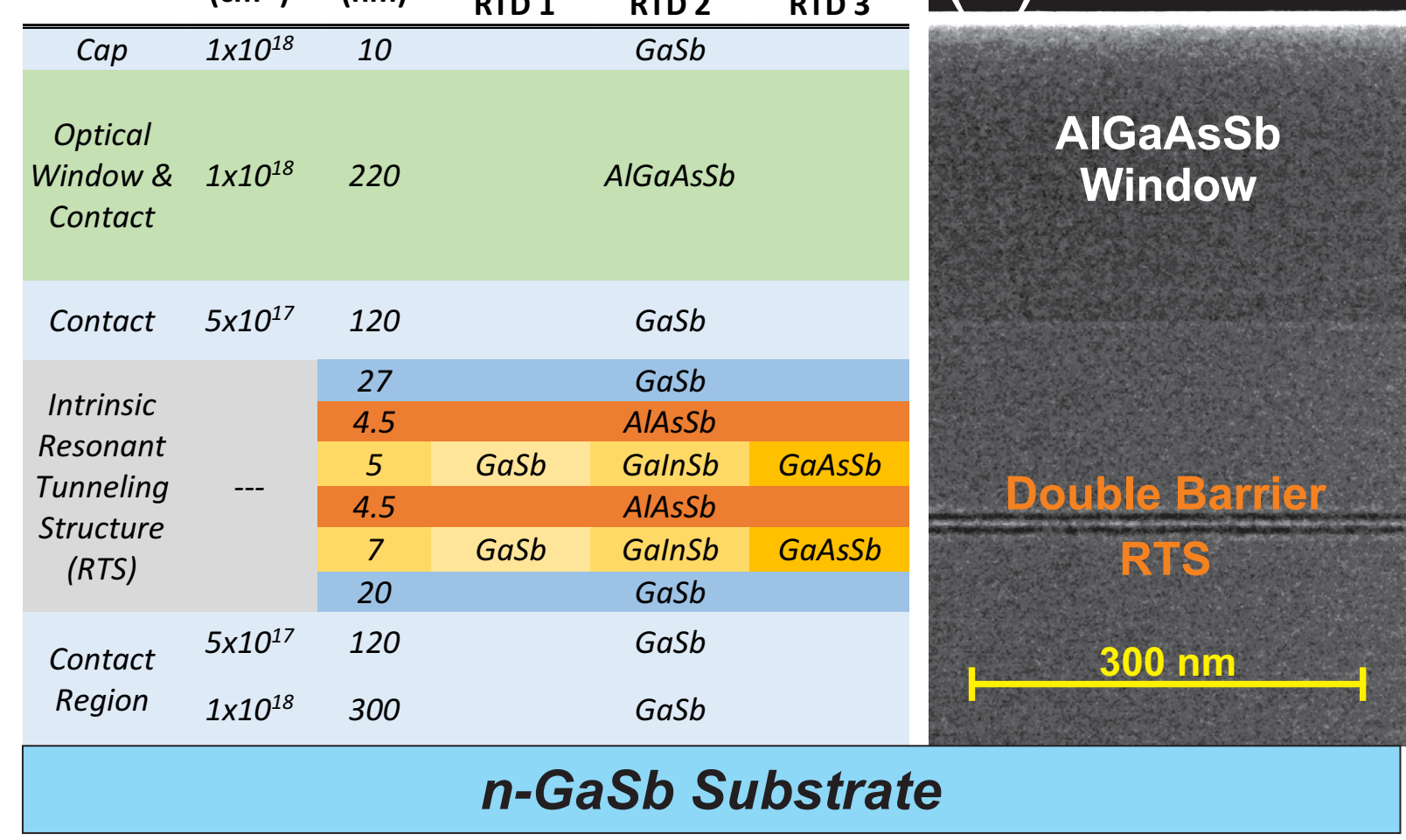

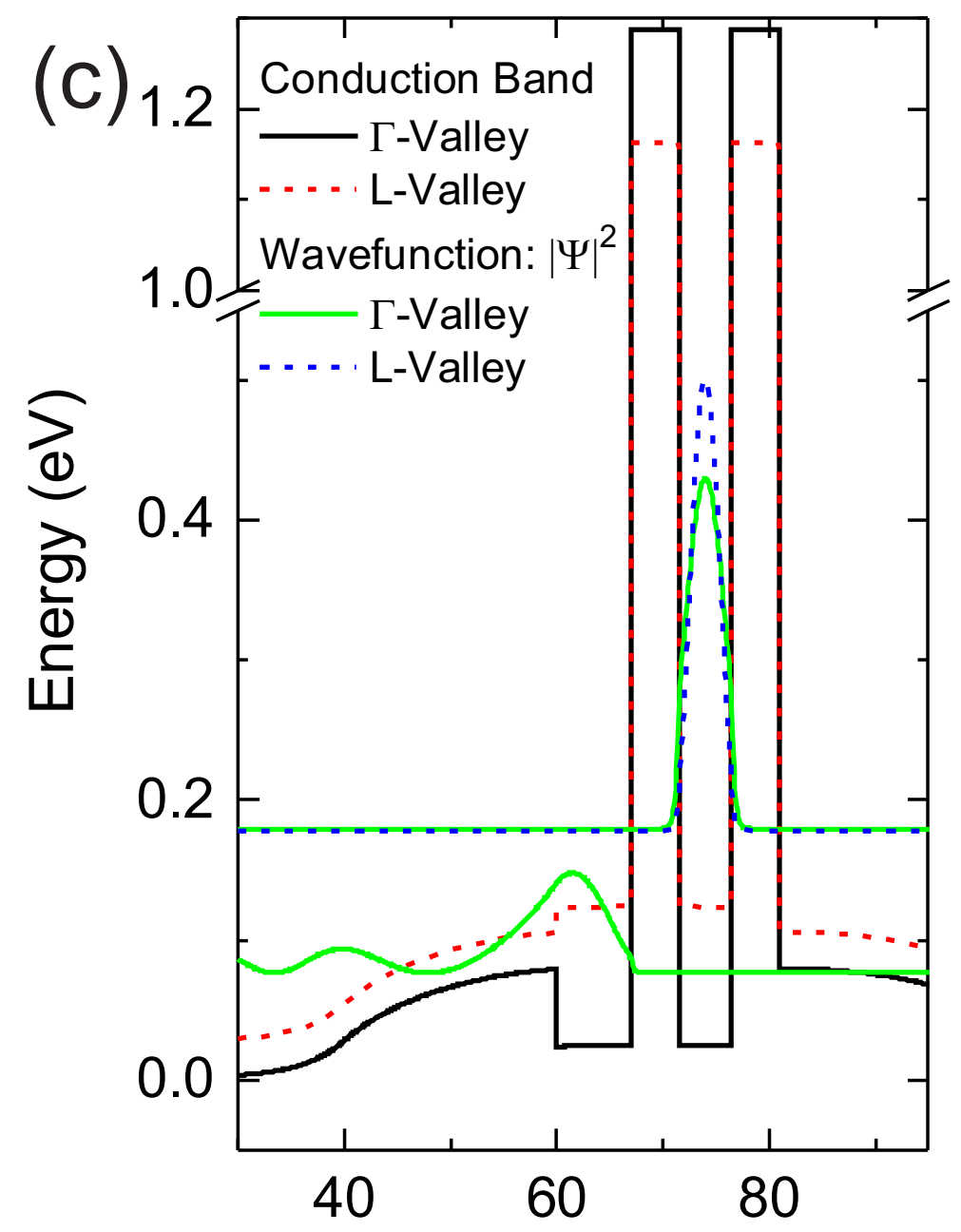

Growth Direction (nm)

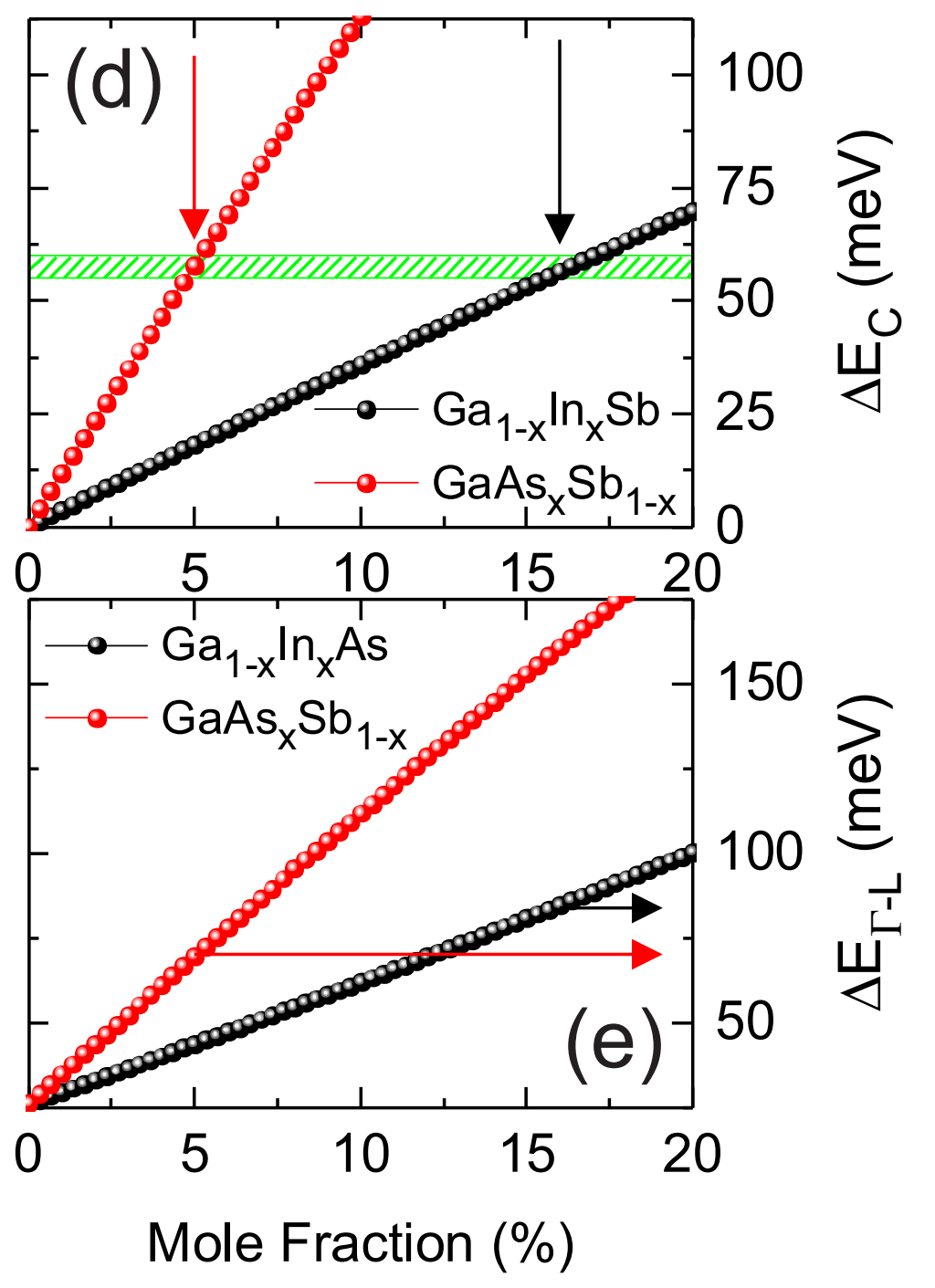



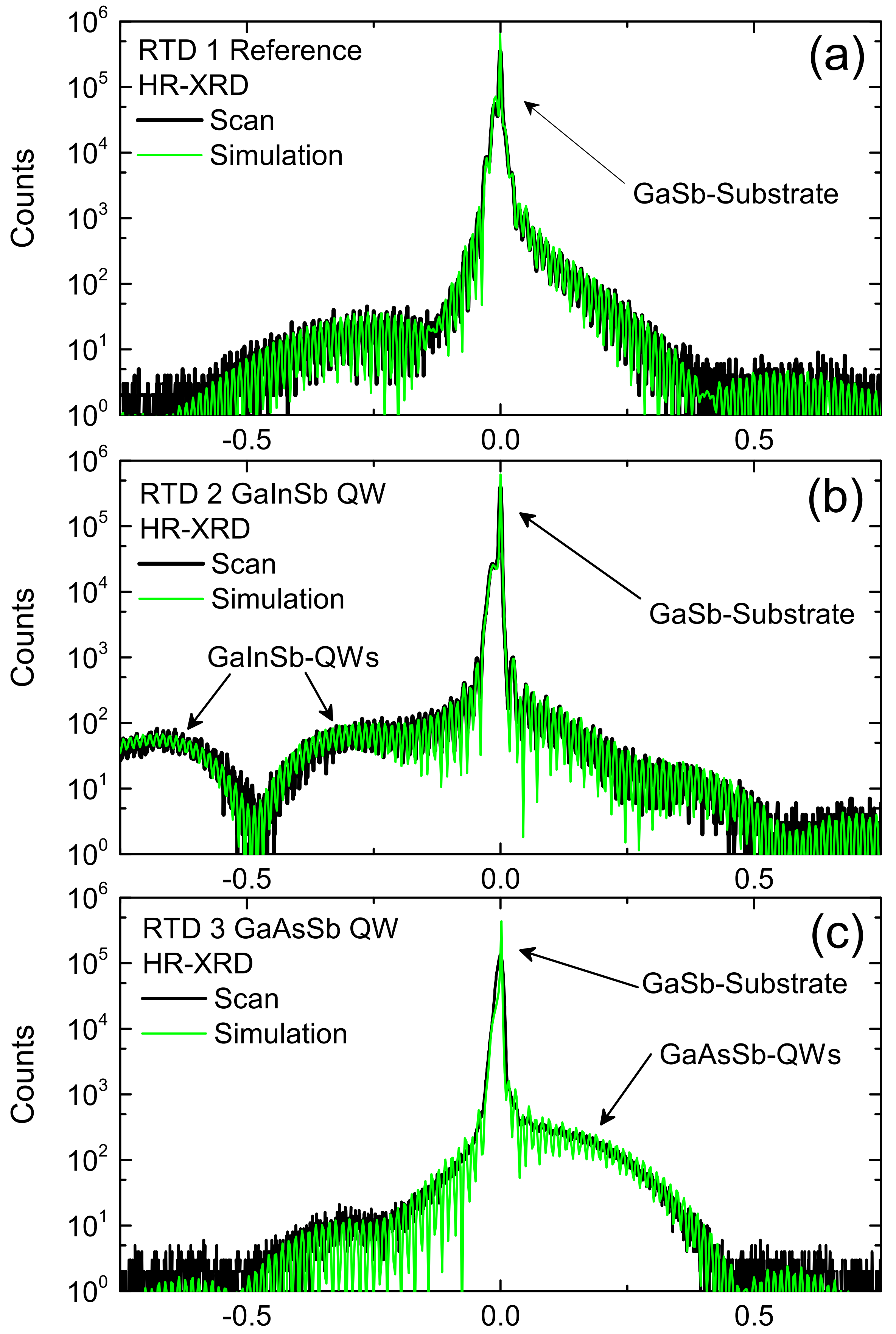

$\Delta \omega$ (degree) 
RTD 2 GalnSb QWs:

$\because \mathrm{T}=77 \mathrm{~K}, \quad \mathrm{PVCR}=7.8 ; \ldots \mathrm{T}=300 \mathrm{~K}, \mathrm{PVCR}=1.4$
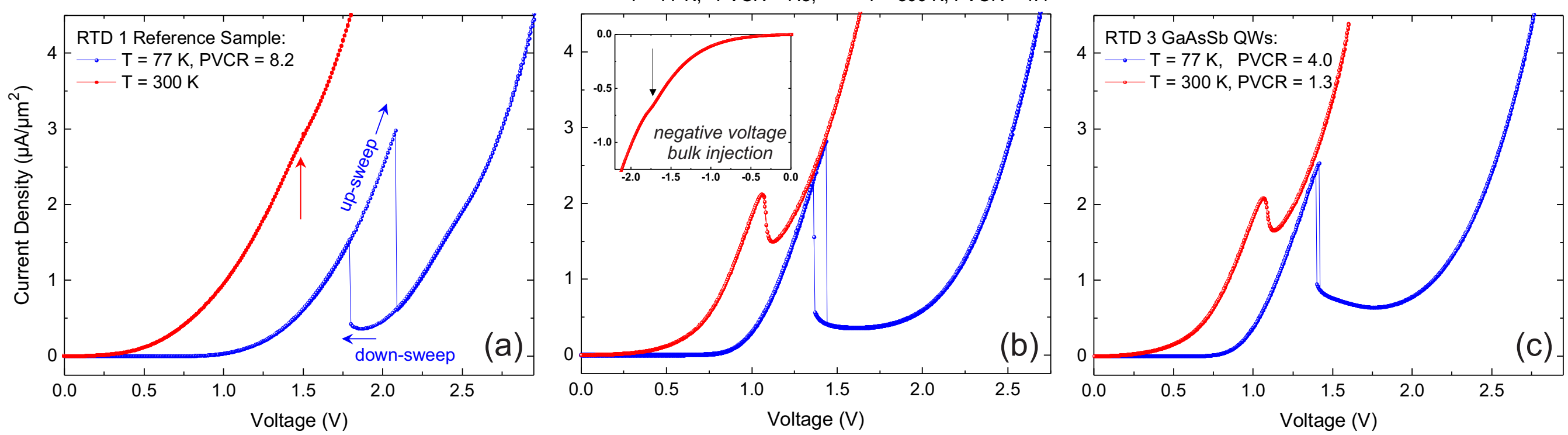
